\title{
An electronic visual stimulator
}

\author{
ROBERT SHAPLEY and MICHELANGELO ROSSETTO \\ Rockefeller University, New York, New York 10021
}

\begin{abstract}
A group of circuits is deseribed that produce various patterns on an oscilloscope screen that are useful in studying vision. These patterns are: a flashing bar, a drifting bar, a flashing grating, and a drifting grating. The circuits have controls for: contrast, velocity, width, position, and orientation with respect to the vertical. This article describes the principles of design and use of these circuit modules as a precise electronic visual stimulator.
\end{abstract}

One can analyze the functional connections in the visual pathway through the study of responses of single visual neurons or psychophysical observers to a multiplicity of visual patterns. The flexibility and precision of the methods used to produce these patterns to a great extent determine the success of this attempt. With this in mind, we have designed and built a set of analog function modules to control an oscilloscope display. This assembly constitutes a versatile and precise electronic visual stimulator.

Throughout the design of these modules we attempted to use integrated circuits (ICs) as much as possible in order to minimize expense and to ease repairs. Several of these ICs have only recently become available. Design of the circuits usually amounted to laying out a block diagram with the ICs and then attending to proper component values, voltage levels, etc. We feel that the block diagrams are of most general interest and so have drawn the figures for this paper accordingly. Anyone wishing detailed schematics should request them directly from us.

There are four basic patterns produced by the electronic display: (1) a flashing bar, (2) a drifting bar, (3) a flashing grating, and (4) a drifting grating. These patterns will be described in more detail. The flashing bar is a stationary rectangular uniform pattern which is either brighter or darker than the background of the screen. The height of the bar is the height of the screen but its width should be controllable, as should its position on the oscilloscope screen. Its intensity is modulated in time. The drifting bar is a bar of fixed intensity, either brighter or darker than the background, the position of which is time varying. A grating is a periodic sequence of bars with a particular intensity protile, e.g., a sinusoidal grating is formed by a periodic spacing of bars with a sine wave profile. A flashing grating is one which is modulated in time so that the intensity at each point of the grating is multiplied by the same temporal modulation factor-in other words, a standing wave. A drifting

This work was supported by Grant No. BMS72-02435 A02 from the National Science Foundation. grating is one in which the bars of the grating are moving in one direction at a uniform velocity. i.e.. a traveling wave. With this basic group of patterns, it is possible to characterize spatial summation and dynamic characteristics of visual neurons in great detail and also to probe the mechanisms of human pattern perception.

One must be able to control some crucial features of these patterns. Particularly important are: (1) contrast or depth of modulation, (2) velocity of drift or temporal frequency of modulation. (3) width of bar or spatial frequency of grating, and (4) position on the screen. Another critical feature is (5) the orientation of these patterns with respect to the vertical. Control of these five critical stimulus parameters was an important consideration in the design of the electronic display circuits described below.

We will first describe the circuit of each module, and a typical range of outputs. A description of the wiring combinations necessary to produce flashing or drifting bars or gratings follows.

\section{RASTER GENERATOR}

The raster generator produces the $\mathrm{X}$ and $\mathrm{Y}$ deflections of the electron beam on the oscilloscope screen so that the screen is uniformly filled with light. It is made up of two main circuits: the line generator and the sweep generator.

The line generator circuit produces a high-frequency (100-200 KC) triangle wave of variable amplitude. symmetric about $0 \mathrm{~V}$. The circuit is diagrammed in Figure 1. An integrated circuit (IC), the XR-205 (EXAR Integrated Systems, Sunnyvale, California) is used to generate the triangle. The frequency of the triangle wave is set by the timing capacitor C. Typical output of the line generator is shown in Figure 1.

The XR-205 IC was selected for the line generator because it had good linearity in the $100-200 \mathrm{kHz}$ range and it contained its own output buffer amplifier. Other chips are available for high-frequency triangle wave generation, but several of the ones we tried began to show unacceptable nonlinearity already at $100 \mathrm{kHz}$, even though the manufacturers claim they work up to 
LINE GENERATOR

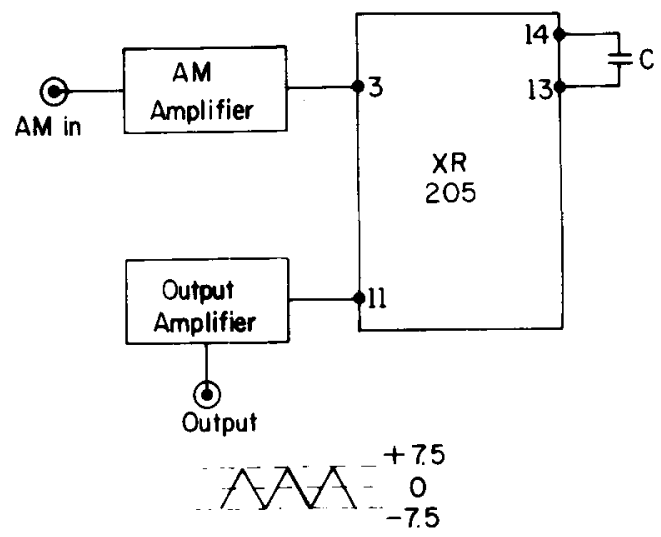

Figure 1. Line generator. Built around the XR 205 IC, this circuit produce a bigh-frequency triangle wave. The frequency of the triangle is set by $\mathrm{C}$, the timing capacitor. There is an amplitude modulation control (AM) which can be used in other applications.

$1 \mathrm{MHz}$. Apparently, $1 \mathrm{MHz}$ is the frequency at which these ICs fail to work at all, but their waveform deteriorates well before this point. The XR-205 cost $\$ 16$ in small quantitites, which is expensive for a chip. One cheaper acceptable replacement is the Signetics NE 566.

The sweep generator is more complicated. This complexity is caused by the need for synchronization of the sweep with external oscillators in order to produce standing patterns on the oscilloscope screen. The circuit for the sweep generator is shown in Figure 2. The building block of this circuit is another IC, the XR 320. The output of the sweep generator is SWEEP OUT, a sawtooth wave with a width determined by the WIDTH dial. The range of widths is $2-10 \mathrm{msec}$ (repetition rates of $100-500 \mathrm{~Hz}$ ). The XR 320 also puts out a positive pulse which, after inversion, can be used

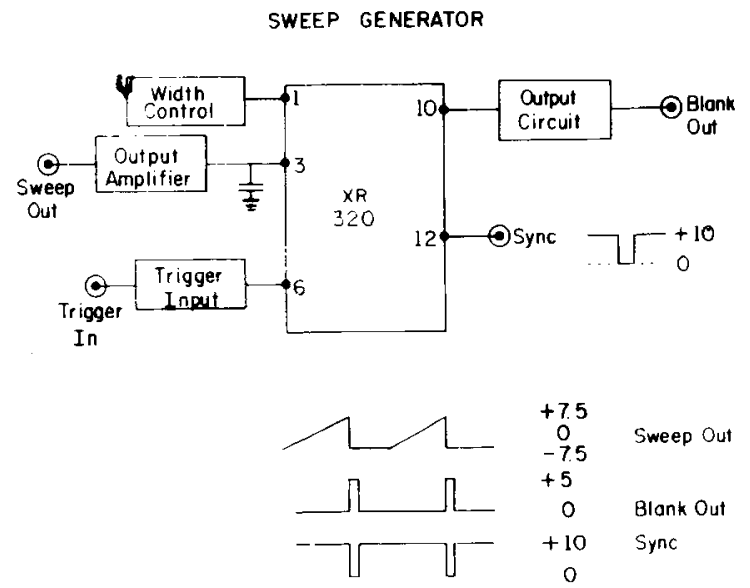

Figure 2. Sweep generator. This circuit uses the XR 320 to generate a sawtooth and two palse outputs, one for blanking (after inverion), and one for syachronization of the pale generator. for blanking the $\mathrm{Z}$-axis during retrace of the sweep; this output is labeled BLANK OUT Sometimes an external oscillator is used to trigger the sweep through the TRIGGER IN input ( $+5 \mathrm{~V}$ for trigger). Under those conditions, the TRIGGER/FREE RUN switch is left in the TRIGGER position. The sweep circuit is nonretriggerable during the sweep duration, set by the width dial. At other times, the sweep is the master oscillator for the display, and the TRIGGER/FREE RUN switch is in the FREE RUN position. In the latter mode, the XR 320 retriggers itself after each cycle.

The XR 320 is a very useful IC for generating linear sweeps up to frequencies of $50 \mathrm{kHz}$. The self-triggering, reset, positive, and negative triggering features made this circuit ideal for a sweep and also as a one-shot for use in the pulse generator. It only cost $\$ 2.25$ in small quantities.

\section{PULSE GENERATOR}

This is the circuit used for making bars on the screen. It consists of two XR 320 timing ICs, an operational amplifier, a comparator, and a specialized output circuit as shown in Figure 3. The purpose of XR 320 \#2 timing circuit is to generate the output pulse. The purpose of the other XR $320 \# 1$ is to generate the delay from a synchronizing pulse. The synchronizing pulse usually comes from the sweep generator described above. Thus, the pulse output is synchronized to the start of the sweep. The delay is voltage controlled by adding a fixed voltage determined by the DELAY dial pot with an external (possibly time varying) voltage brought in at the DELAY IN socket. The summed voltage is led to one side of the comparator which compares this "threshold" with a ramp generated by the XR 320 \#1. When the ramp crosses the "threshold," the comparator changes state very rapidly. This resets the XR 320 \#1, and the resetting produces an output edge from the XR 320 \# 1 which triggers the XR 320 \#2. Capacitors and resistors connected to the XR 320s determine the range of delay and pulse widths. The output circuit is wired so as to give symmetrical outputs (5 V) of opposite polarity at the + and - output sockets and $0 \mathrm{~V}$ when the pulse is off. There is also a TRIGGER output for triggering other pulse generators of this type.

\section{DRIFTING GRATING GENERATOR}

This circuit is used to generate sinusoidal and square wave gratings, either drifting or stationary. It consists of two commercially available sine-cosine oscillators (Models 441 and 444 from Frequency Devices, Haverhill, Mass.), a sine-cosine potentiometer, a pair of multipliers, three operational amplifiers, a comparator circuit, and an automatic gain control circuit. These various circuits are shown in Figure 4. The principle of the device is to form the sum 


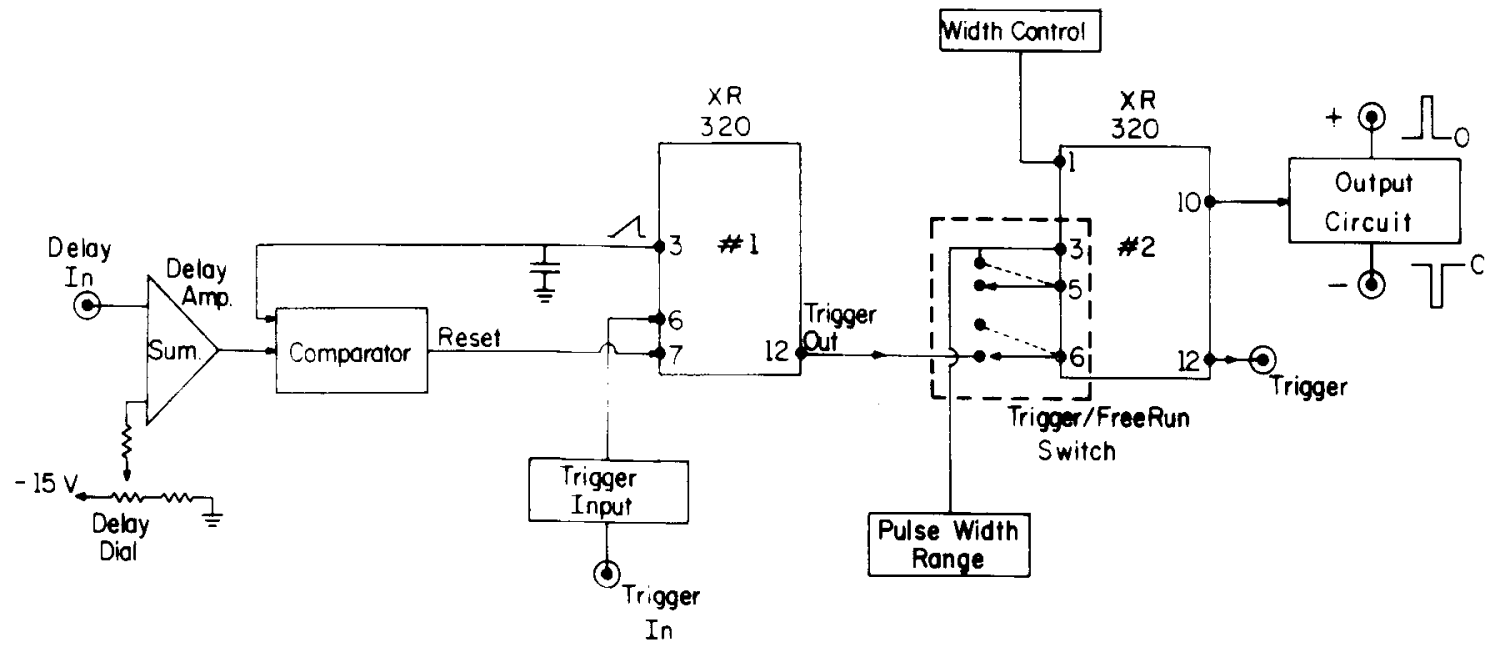

Figure 3. Pulse generator. This circuit uses two XR 320 timing circuits, one for generating the output pulse, the other for producing a voltage-controlled delay with respect to the start of the sweep. See text for detailed description.

$\sin \left(2 \pi f_{1}\right) \cos \left(2 \pi f_{2} t\right)+\cos \left(2 \pi f_{1} t\right) \sin \left(2 \pi f_{2} t\right)$ which equals $\sin \left[2 \pi\left(f_{1}+f_{2}\right) t\right]$, where $f_{1}$ is the frequency (in CPS) of the low-frequency oscillator, and $f_{2}$ is the frequency of the high-frequency oscillator. When this composite wave form is used to trigger the sweep generator, and then $\sin \left(2 \pi \mathrm{t}_{2} \mathrm{t}\right)$ is connected to the $\mathrm{Z}$ axis of the oscilloscope, the circuit will produce on the screen a sinusoidal grating drifting to the right at a frequency of $f_{1}$ bars $/$ sec. The circuit in Figure 4 actually forms $\sin \left[2 \pi\left(f_{2} \pm f_{1}\right) t\right]$ where the sign of the $f_{1}$ term is determined by the LEFT/RIGHT switch. ${ }^{1}$

In place of the low-frequency oscillator, one can switch in the $\sin / \cos$ potentiometer with the F1 PHASE switch. In PHASE position, the output of the module is $\sin \left(2 \pi \mathrm{f}_{2} \mathrm{t}+\varnothing\right)$, where $\varnothing$ is the angle of shaft of the $\sin / \cos$ potentiometer. This can be used to

\section{DRIFTING GRATING GENERATOR}

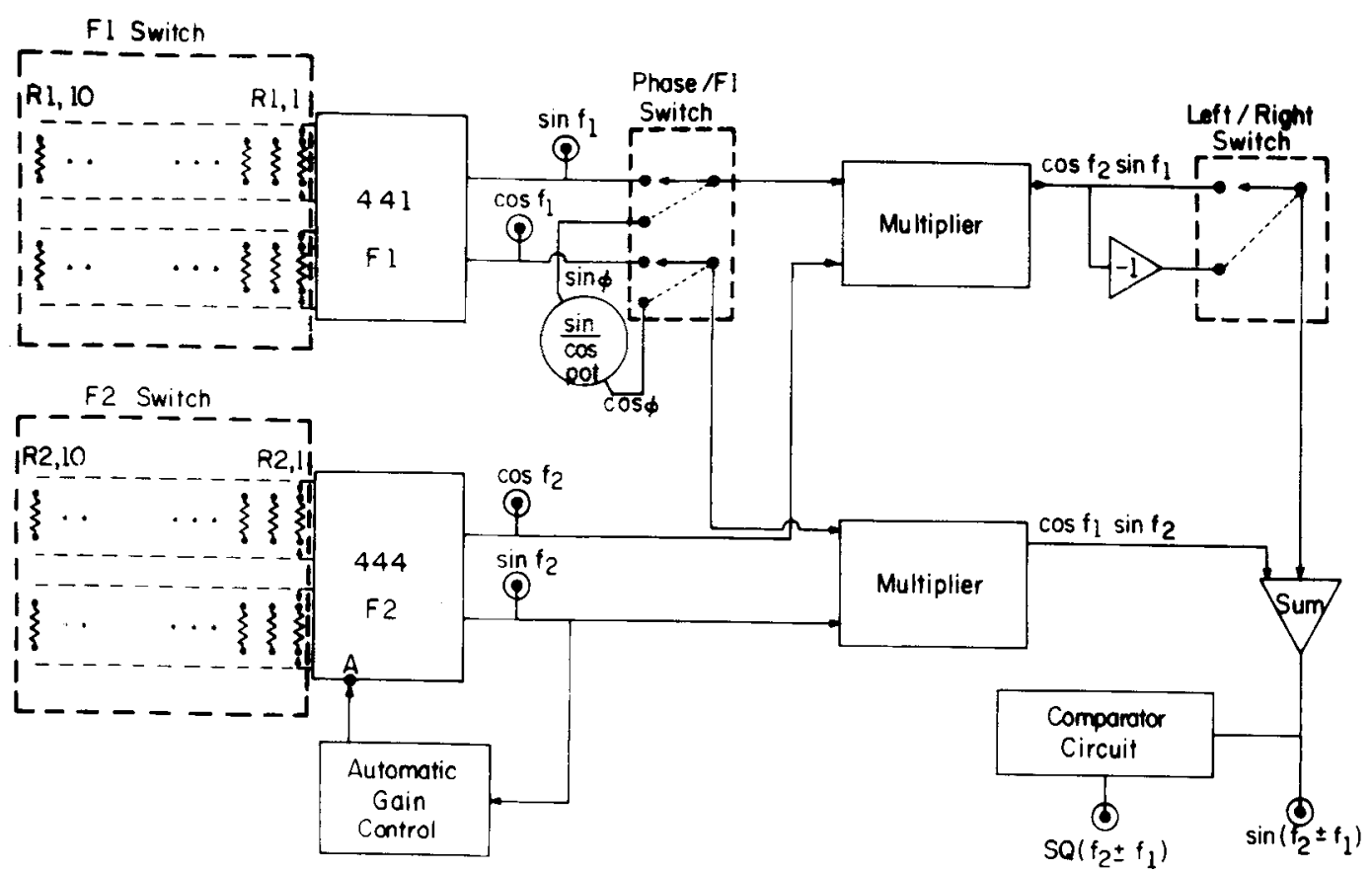

Figure 4. Drifting grating generator. Built around the two quadrature oscillators, the Frequency Devices 441 and 444 , this module produces stationary or drifting sinusoidal or square wave gratings. 


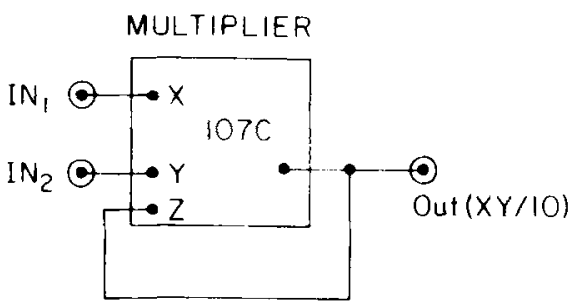

SUMMING AMPLIFIER

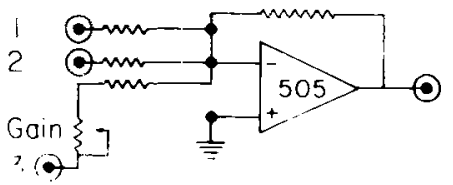

Figure 5. Muttiplier and summing amplifier. The multiplier was the Hybrid Syotems 107C and was wired as apecified by the manufacture. Trimpots on gabs and balance inputs were abo added althongh not drawn in this echematic. The summing amplifier was the high-speed AD 505. There were three inputs, two unity galn and one connected for variable gain. atually blanking from the sweep generator went mto Input 1 and $Z$ axts modulation went into Input 2.

change the spatial phase, in other words the position, of the grating formed by $\sin \left(2 \pi f_{2} t\right)$ on the screen.

The resistor pairs $R_{1}, 1$ through $R_{1}, 10$ control the frequencies of Oscillator 1 (temporal frequency) and $R_{2}, 1$ through $R_{2}, 10$ the frequencies of Oscillator 2 (spatial frequency), as indicated in the figure.

The quadrature oscillators made by Frequency Devices are not integrated circuits but rather solid state function modules. They are rather large and expensive, at about $\$ 100$ each. But these disadvantages are outweighed by the advantages: quadrature outputs, good waveform purity, ease of frequency selection with resistive programming, and a wide frequency range of a factor of 50 .

\section{MULTIPLIER AND SUMMING CIRCUIT}

Diagrams of these circuits are shown in Figure 5.

In order to get flashing patterns, we used an analog multiplier (107C, Hybrid Systems, Burlington, Mass.) wired as recommended by the manufacturer. This multiplier allows $1 \%$ accuracy or better and was quickly available. There are now many competitive monolithic circuits which do the same job, e.g., Analog Devices 530, Burr-Brown 4203 or Intronix 540 for about the same price ( $\$ 40$ per). The output of the multiplier is $X Y / 10$, where $X$ and $Y$ are the two inputs in units of volts, with a maximum allowable input of 10. Either the output of the pulse generator or output of the drifting grating generator was led to one input of the multiplier and a slow modulation signal was led to the other input. The modulation signal could be derived from commercial function generators or from the D/A output of a digital computer.
The summing amplifier, an Analog Devices 505, was used to add the blanking signal, from the sweep generator, to the output of either the multiplier, or the pulse generator, or the drifting grating generator. Then the output of the summing amplifier was connected to the $\mathrm{Z}$ axis of the oscilloscope for display. The 505 operational amplifier is extremely fast ( 800 nsec to $.1 \%$ settling time), stable, and easy to use. Competitive amplifiers on chips are available, but since it only costs $\$ 15$, and is so good, it is worth using.

\section{ROTATOR}

To study single cells in the visual cortex, control of the orientation of patterns on the screen is essential. The rotator circuit, diagrammed in Figure 6, performed this task. The principle was that coordinate position is transformed as follows, where the rotation angle is $\theta$.

$$
\begin{aligned}
& x^{\prime}=x \cos \theta+y \sin \theta \\
& y^{\prime}=-x \sin \theta+y \cos \theta
\end{aligned}
$$

$\operatorname{Sin} \theta$ and $\cos \theta$ were derived from a $\sin / \cos$ potentiometer (or from $2 \mathrm{D} / \mathrm{A}$ outputs of a digital computer, fed through Sockets $S$ and $C$ ) and multiplied with the sweep generator output $(X)$ and the

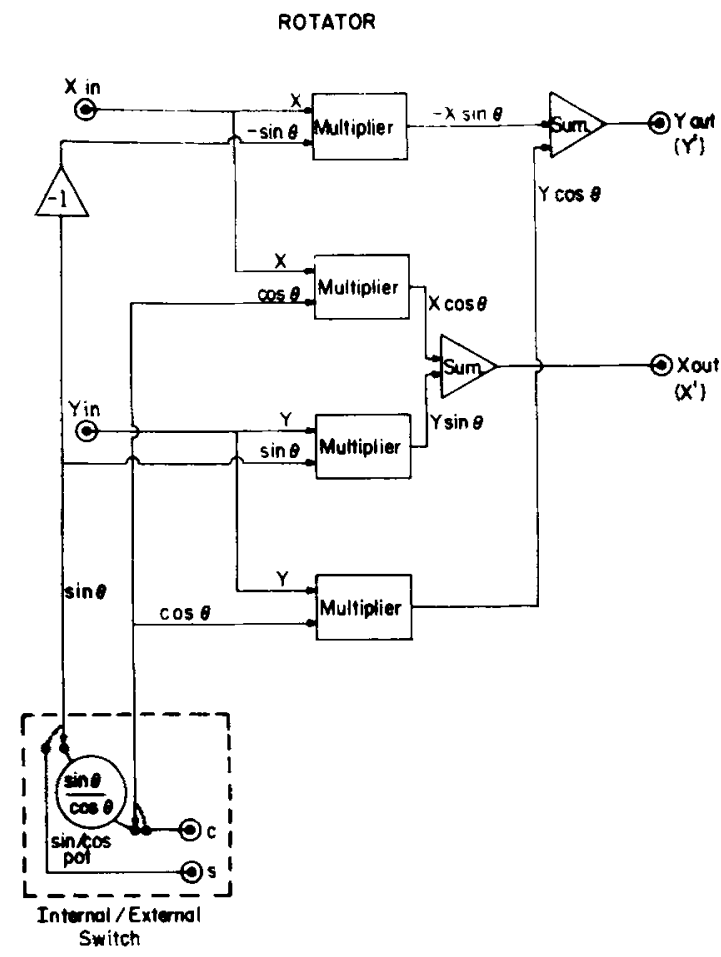

Figure 6. Rotator. This circuit rotates the raster, and thereby the pattern, on the oucllowcope sereen. It uses a sin/cos potentiometer, or two external inpots in quadrature, to set the orientation angle of the raster with respect to the vertical. $Y_{\text {tn }}$ comes from the line generator, $X_{\text {in }}$ comes from the sweep generator. 
line generator output (Y). The multipliers used were monolithic Burr-Brown $4203 \mathrm{~K} 1 \% 1 \mathrm{MHz}$ smallsignal-bandwidth multipliers. The four products were combined in two pairs by addition in the two high-speed Analog Devices $\mathbf{5 0 5}$ operational amplifiers to give $X^{\prime}$ and $Y^{\prime}$.

The $\mathrm{X}^{\prime}$ and $\mathrm{Y}^{\prime}$ signals were fed into $5 \mathrm{~A} 15$ plugins of the Tektronix $5103 \mathrm{~N}$ oscilloscope used for the display. In any application involving rotation, it is essential that the $X$ and $Y$ amplifiers of the scope be balanced and have little phase shift at least up to the frequency of the line generator.

The external $S$ and $C$ inputs allow patterns to be rotated by time-varying signals, as long as these inputs are related as sine to cosine.

The rotator circuit is the one which puts most demands on the available ICs. In this circuit, the multipliers were about as fast and accurate as one can find. and the operational amplifiers are the fast AD 505s. Phase shift and inaccuracy in the multipliers may cause the pattern to be degraded so it pays to get the good multipliers even though they cost $\$ 40$ each. It is also important to have balanced horizontal and vertical deflection amplifiers on the display itself. Furthermore. the $\mathrm{X}$ and $\mathrm{Y}$ in puts to the rotator ought to be of equal peak to peak amplitude and symmetrical about $0 \mathrm{~V}$. This is required in order to achieve rotation about the midpoint of the pattern.

The total cost for parts for the circuits described above was $\$ 1.200$, including also the cost of a power supply. Most of the circuit components were not major contributors to this cost, with the exception of the multipliers in the drifting grating generator and the rotator, and the quadrature oscillators in the drifting grating generator. Potentiometers used to control circuit parameters also contributed substantially to the total cost.

\section{EXAMPLES}

We will now describe how to connect the individual circuits to produce four basic visual patterns. In describing the stimulus parameter ranges below, we have assumed a distance from the screen to the eye of $57 \mathrm{~cm}$, so that the 5100 oscilloscope subtends $8 \mathrm{deg} \mathbf{x}$ $10 \mathrm{deg}$ and $1 \mathrm{~cm} \equiv 1 \mathrm{deg}$ visual angle.

Although it is possible to do it differently, we have in general used the square wave output of the drifting grating generator as a master oscillator, with the sweep generator triggered off of it.

In all patterns, the $X$ and $Y$ inputs to the oscilloscope deflection amplifiers are from the rotator which in turn always receives its $X$ input from the sweep generator and its $\mathrm{Y}$ from the line generator. The only difference between patterns is in which waveform is connected to the summing amplifier which feeds the $\mathrm{Z}$ axis of the oscilloscope. The connections for the different patterns are as follows: (1) Flashing bar. The pulse generator output goes to one multiplier input. Some external waveform generator (e.g., a Wavetek or a D/A converter from a computer) is fed into the other multiplier input. The multiplier output goes to the summing amplifier. The position of the bar on the screen is set by the DELAY dial on the pulse generator. and can be modified by a voltage fed into the DELAY IN socket. Bar widths from .1 deg visual angle to $10 \mathrm{deg}$ can be produced. (2) Drifting bar. The drift rate and extent are controlled by a time-varying voltage fed into the DELAY IN socket of the pulse generator; back and forth motion is produced by a triangle wave input, unidirectional motion with a sawtooth input. and quick jumps with a square wave input. Drift velocity can be varied from 0 to $125 \mathrm{deg} / \mathrm{sec}$.

The pulse generator output goes through an attenuator (for contrast control) and thence to the summing amplifier. The attenuator is a Hewlett-

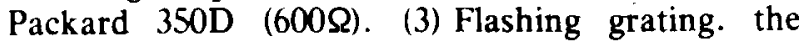
connections are the same as for the flashing bar except that sine wave is substituted for pulse in one of the two multiplier inputs. The drifting grating F1/PHASE switch is set to PHASE, and spatial phase of the grating is then adjustable by the dial. Either sin $\left(2 \pi f_{2} t\right)$ or $\cos \left(2 \pi f_{2} t\right)$ is connected to one multiplier input, and a slow modulation signal to the other multiplier input. Spatial frequency of the grating is controlled by the 10 position switch marked F2. Multiplier output goes to the summing amplifier in put and thence to scope $\mathrm{Z}$ axis.

Flashing gratings we have used have been of two main types. An on-off grating is produced by using a rectangular wave which goes between $0 \mathrm{~V}$ and some positive voltage, say $5 \mathrm{~V}$. When this modulation signal is $0 \mathrm{~V}$, the screen of the oscilloscope is blank at the mean luminance. When it is $+5 \mathrm{~V}$, the gratings are present at full contrast. The other sort of pattern is an alternating phase (or contrast reversal) grating. To create this pattern, one uses a modulation signal going between $-5 \mathrm{~V}$ to $+5 \mathrm{~V}$, so as to shift the spatial phase of the grating by $180 \mathrm{deg}$ (multiply by-1) at the repetition rate of the modulation. The modulation signal can be square wave or sinusoidal, to give abrupt or smooth alternation, respectively. (4) Drifting grating. As with the drifting bar, the output of the drifting grating generator is connected through an attenuator to the summing amplifier and in turn to the $Z$ axis of the oscilloscope. Spatial and temporal frequencies are controlled by the $F 2$ and $F 1$ switches, respectively. Direction of motion is determined by the LEFT/RIGHT switch. The drift rate in bars/second is independent of spatial frequency. This means that drift velocity in centimeters/second on the screen is inversely proportional to spatial frequency. In many 
visual experiments, it is better to keep the drift rate constant rather than drift velocity. Drift rates from .5 to $25 \mathrm{~Hz}$ were achieved. while spatial frequencies ranged from .2 cycles/deg up to 25 cycles/deg. Drift velocities therefore ranged from $.02 \mathrm{deg} / \mathrm{sec}$ up to $125 \mathrm{deg} / \mathrm{sec}$.

Contrast was limited to a maximum of $60 \%$ without distortion; this limitation was set by the oscilloscope $\mathbf{Z}$ axis amplifier rather than by our circuits.

\section{NOTE}

1. The idea for the drifting grating circuit. i.e. forming $\sin \left[2 \pi\left(f_{2}+f_{1}\right) t\right]$ by the trigonometric identity, was suggested by Dr. J. G. Robson of the physiological Laboratory. Cambridge. England.

(Received for publication July 28, 1975; revision received November 20,1975 .) 